\title{
Special issue on dynamics of socioeconomic systems: systemic risk-finance
}

\author{
Philippe de Peretti ${ }^{1} \cdot$ Bice Cavallo ${ }^{2} \cdot$ Biagio Simonetti $^{3} \cdot$ Massimo Squillante $^{3} \cdot$ Jorgen Vitting-Andersen $^{4}$
}

Published online: 8 May 2020

(C) Springer-Verlag GmbH Germany, part of Springer Nature 2020

Over the past decades, complexity has emerged as an alternative way to understand and model complex dynamical phenomena in a number of fields. Instead of focusing on local and independent behaviors, complexity suggests that observed phenomena are the results of complex interactions between components of a system. The DySES conference, which took place at the Sorbonne in 2018, gave the unique opportunity to present the state of the art in the complexity field and in many areas including the socioeconomic ones. This special issue of Soft Computing presents papers in finance both in the field of complexity and beyond it. Many topics are covered. Jay et al. focus on portfolio management and introduce robust covariance matrices using recent developments in the random matrix theory (RMT). Levantesi et al. propose a novel approach, combining the random forest and the $2 \mathrm{D}$ $\mathrm{P}$-spline in order to provide a more accurate mortality rate forecasting, therefore extending the Lee-Carter model. Chorro et al. focus on option pricing. They start from the inverse Gaussian GARCH model and build a new pricing kernel. Cerqueti et al. work on complex networks and introduce new influence measures based on vertex centrality. They apply their measure to the SP100 universe. Still within the complexity field, Gatfaoui et al. introduce a test of non-chaoticity when data are noisy. They show that financial data do not have a chaotic behavior. Fiori et al. build a model to capture systemic risk. In particular, they set the focus on the inequalities of wealth and income, using the well-known Gini coefficient. Di Paolo tackles the problem of the increase in life expectancy with regard to pension funds and annuity providers. The concept of observed survival probabilities is extensively used. Within a related area, D'Amato et al. propose a de-risking strategy model for LTC insurers that face demographic changes implying disability risks. They suggest new methodologies. Baione et al. consider non-life risk premium and study the diversification effect. They use quantile regressions to estimate the individual conditional loss. Kaucic et al. study investment strategies known as enhanced indexing. They introduce an improved version of the particle swarm optimization algorithm (PSO) and present an example using the Euro Stoxx. At last, Kudlàk et al. question the importance of dedicated local budgets concerning crisis management. As guest editors, we would like to thank Soft Computing for giving us the opportunity to have this special issue done on all these topics.

Publisher's Note Springer Nature remains neutral with regard to jurisdictional claims in published maps and institutional affiliations.

\footnotetext{
All the authors contributed equally to this work.

Philippe de Peretti

Philippe.de-Peretti@univ-paris1.fr

University of Paris 1 Panthéon-Sorbonne, Paris, France

University of Naples Frederico II, Naples, Italy

3 University of Sannio, Benevento, Italy

4 CNRS, University of Paris 1 Panthéon-Sorbonne, Paris, France
} 